Alberta Thy-25-92

August 1992

\title{
On the QCD corrections to the charged Higgs decay of a heavy quark.
}

\author{
Andrzej Czarnecki* and Sacha Davidson** \\ Department of Physics, University of Alberta, Edmonton, Canada T6G 2J1
}

\begin{abstract}
Using dimensional regularization for both infrared and ultraviolet divergences, we confirm that the QCD corrections to the decay width $\Gamma\left(t \rightarrow H^{+} b\right)$ are equal to those to $\Gamma\left(t \rightarrow W^{+} b\right)$ in the limit of a large $t$ quark mass.
\end{abstract}


Many extensions of the Standard Model contain more than the one necessary Higgs doublet, and the new degrees of freedom appear as extra Higgs scalars, some of which are charged (see [1] and references therein). We consider here the decay $t \rightarrow H^{+} b$ in a model with two Higgs doublets. The first order QCD correction to this process has been calculated by two groups [1] 3] who disagree with each other. In this letter we present a calculation of this correction in the limit of a very heavy top quark, i.e., neglecting the masses of the bottom quark and the Higgs boson. The effect of finite $m_{H}$ and $m_{b}$ will be addressed in a forthcoming paper. We use dimensional regularization to cope with both the ultraviolet and infrared divergences, which greatly simplifies the calculation, especially the real radiation part.

We take $H_{1}$ and $H_{2}$ to be the doublets whose vacuum expectation values respectively give masses to the down and up type quarks. The physical charged Higgs $H^{+}$is a linear combination of the charged components of $H_{1}$ and $H_{2}$, so if we neglect all the Yukawa couplings except that of $H_{2}$ to the third generation $=h_{t t}^{(2)}$, the top only couples to the $H_{2}$ component of $H^{+}$. The interaction Lagrangian relevant to the decay $t \rightarrow H^{+} b$ is then:

$$
\begin{aligned}
\mathcal{L} & =h_{t t}^{(2)} \cos \beta V_{t b} H^{+} \bar{t}\left(\frac{1-\gamma_{5}}{2}\right) b+h . c . \\
& =\frac{g}{2 \sqrt{2} m_{W}} V_{t b} \cot \beta m_{t} H^{+} \bar{t}\left(1-\gamma_{5}\right) b+\text { h.c. }
\end{aligned}
$$

where $H^{+}=\cos \beta H_{2}^{+}-\sin \beta H_{1}^{+}$and $\cot \beta=\left\langle H_{1}\right\rangle /\left\langle H_{2}\right\rangle$ is the ratio of vacuum expectation values of the two Higgs doublets.

In the following calculations we take the space-time dimension to be $D=4-2 \epsilon$. The mass of the decaying quark is taken to be the renormalization mass scale, and we also use it as a unit of energy: $m_{t}=1$. In the limit of a very heavy top quark the above interaction leads to the tree-level decay rate:

$$
\Gamma^{(0)}\left(t \rightarrow H^{+} b\right)=\frac{G_{F}}{\sqrt{2}} \frac{\Gamma(1-\epsilon)}{2^{3-2 \epsilon} \pi^{1-\epsilon} \Gamma(2-2 \epsilon)} \cot ^{2} \beta\left|V_{t b}\right|^{2} \rightarrow \frac{G_{F}}{8 \sqrt{2} \pi} \cot ^{2} \beta\left|V_{t b}\right|^{2} .
$$

The first order QCD corrections to this formula arise due to virtual gluon exchange and radiation of a real gluon. We first deal with the virtual gluon correction to the vertex $t H^{+} b$. In the limit $m_{b}=m_{H}=0$ the spinor structure of this vertex 
remains unchanged and the unrenormalized correction amounts to the multiplication of the tree level rate by a factor

$$
\Lambda=C_{F} g_{s}^{2} \frac{\Gamma(1+\epsilon)}{(4 \pi)^{\frac{D}{2}}}\left(-\frac{1}{\epsilon^{2}}+\frac{2}{\epsilon}\right) .
$$

where the colour factor $C_{F}$ is $4 / 3$ for $S U(3)$. The counterterm for this vertex involves the wave function and mass renormalization constants [4, 1]:

$$
\Lambda_{c . t .}=\frac{1}{2}\left(Z_{2}^{t}-1\right)+\frac{1}{2}\left(Z_{2}^{b}-1\right)-\frac{\delta m_{t}}{m_{t}}-\frac{\delta m_{b}}{m_{b}} .
$$

If we use the same $\epsilon$ to regularize both UV and IR divergencies we obtain for the renormalization constants:

$$
\begin{aligned}
& Z_{2}^{t}-1=-\frac{\delta m_{t}}{m_{t}}=C_{F} g_{s}^{2} \frac{\Gamma(1+\epsilon)}{(4 \pi)^{\frac{D}{2}}}\left(-\frac{3}{\epsilon}-4\right), \\
& Z_{2}^{b}-1=\frac{\delta m_{b}}{m_{b}}=0 .
\end{aligned}
$$

The contribution of the virtual correction to the decay rate is:

$$
\begin{aligned}
\Gamma_{\text {virt }}^{(1)}\left(t \rightarrow H^{+} b\right) & =2\left(\Lambda+\Lambda_{\text {c.t. }}\right) \Gamma^{(0)}\left(t \rightarrow H^{+} b\right) \\
& =\frac{G_{F}}{\sqrt{2}} \cot ^{2} \beta\left|V_{t b}\right|^{2} \alpha_{s} \frac{2^{-3+4 \epsilon} \pi^{-2+2 \epsilon}}{3 \Gamma(2-2 \epsilon)}\left(-\frac{2}{\epsilon^{2}}-\frac{5}{\epsilon}-12-\frac{\pi^{2}}{3}\right)
\end{aligned}
$$

We now turn our attention to the effect of real gluon radiation from the initial or final quark. If we denote the amplitudes for these processes by

$\mathcal{A}_{1}$ and $\mathcal{A}_{2}$ respectively, the contribution of the real radiation to the decay width is:

$$
\Gamma_{\text {real }}^{(1)}\left(t \rightarrow H^{+} b G\right)=\frac{1}{2} \frac{G_{F}}{\sqrt{2}} \cot ^{2} \beta\left|V_{t b}\right|^{2} 4 \pi \alpha_{s} \int d R_{3}(t ; b, H, G)\left|\mathcal{A}_{1}+\mathcal{A}_{2}\right|^{2}
$$

where the coupling constants have been factored out and $t, b, H$ and $G$ denote the fourmomenta of the initial and final quarks, charged Higgs boson and the gluon.

The advantage of using dimensional regularization for the infrared and colinear divergences is that we need not introduce a mass for the gluon and the integration over three body massless phase space is very simple. We choose to parametrise it by the variables $x=2 t \cdot G$ and $z=1-2 t \cdot b$ in terms of which the three body phase space integration becomes: 


$$
\int d R_{3}(t ; b, H, G)=\frac{2^{4 \epsilon-7} \pi^{2 \epsilon-3}}{\Gamma(2-2 \epsilon)} \int_{0}^{1} \frac{d x}{(1-x)^{\epsilon}} \int_{0}^{x} \frac{d z}{z^{\epsilon}(x-z)^{\epsilon}} .
$$

After summing over the polarizations of the $b$ quark and the gluon, and averaging over the polarizations of the $t$ quark, the squares of the amplitudes become:

$$
\begin{aligned}
\left|\mathcal{A}_{1}\right|^{2} & =-\frac{4}{x^{2}}[2(1-x)+x(1-\epsilon)(z-x)] \\
\left|\mathcal{A}_{2}\right|^{2} & =\frac{4 x}{x-z}(1-\epsilon) \\
\mathcal{A}_{1} \mathcal{A}_{2}^{*}+\mathcal{A}_{2} \mathcal{A}_{1}^{*} & =\frac{8}{x(x-z)}[1-x+x(1-\epsilon)(z-x)]
\end{aligned}
$$

The integration over the phase space can be done exactly in any dimension. In the limit $\epsilon \rightarrow 0$ the contribution of the real radiation becomes:

$$
\Gamma_{\text {real }}^{(1)}\left(t \rightarrow H^{+} b\right)=\frac{G_{F}}{\sqrt{2}} \cot ^{2} \beta\left|V_{t b}\right|^{2} \alpha_{s} \frac{2^{-3+4 \epsilon} \pi^{-2+2 \epsilon}}{3 \Gamma(2-2 \epsilon)}\left(\frac{2}{\epsilon^{2}}+\frac{5}{\epsilon}+17-\pi^{2}\right) .
$$

Although the respective phase space integrations of

$$
\left|\mathcal{A}_{1}\right|^{2},\left|\mathcal{A}_{1}\right|^{2} \text { and }
$$

$\mathcal{A}_{1} \mathcal{A}_{2}^{*}+\mathcal{A}_{2} \mathcal{A}_{1}^{*}$ give different results from the analogous amplitudes with a $W^{+}$replacing the charged scalar, their sum nevertheless gives the same total contribution in both processes. This is in agreement with the general argument based on the equivalence theorem in ref. [3].

Finally we add the effects of the virtual and real gluons to obtain the first order QCD correction, so the decay rate (with $m_{t}$ reinstated) becomes:

$$
\Gamma\left(t \rightarrow H^{+} b\right)=\frac{G_{F}}{8 \sqrt{2} \pi} m_{t}^{3} \cot ^{2} \beta\left|V_{t b}\right|^{2}\left[1+\frac{\alpha_{s}}{3 \pi}\left(5-\frac{4 \pi^{2}}{3}\right)\right] .
$$

This is identical to the result obtained in ref. [3] and also to the analogous correction

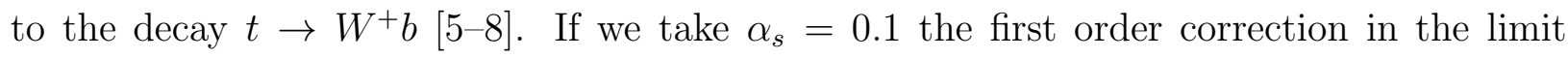
$m_{b}=m_{H}=0$ is approximately equal $-0.87 \%$. This is in disagreement with the value reported in ref. [1, 2].

\section{ACKNOWLEDGMENTS}

We gratefully acknowledge support for our doctoral scholarships: A.C. from the Killam Foundation and S.D. from NSERC. This research was also partially supported by a grant 
to A. N. Kamal from the Natural Sciences and Engineering Research Council (NSERC) of Canada.

This preprint was typeset using REVTEX. 


\section{REFERENCES}

* Bitnet address: USERAPRU@UALTAMTS

** Permanent address after October 1st, 1992: Center for Particle Astrophysics, University of California at Berkeley, Berkeley, California 94720.

[1] C. S. Li and T. C. Yuan, Phys. Rev. D42 (1990) 3088.

[2] C. S. Li et al., Phys. Lett. B285 (1992) 137.

[3] J. Liu and Y. P. Yao, preprint UPR-0508T (1992).

[4] E. Braaten and J.P. Leveille, Phys. Rev. D22 (1980) 715.

[5] M. Jeżabek and J. H. Kühn, Nucl. Phys. B314 (1989) 1.

[6] A. Czarnecki, Phys. Lett. B252 (1990) 467.

[7] J. Liu and Y. P. Yao, Int. J. Mod. Phys. A6 (1991) 4925.

[8] C. S. Li, R. J. Oakes and T. C. Yuan, Phys. Rev. D43 (1991) 3759. 